\title{
Pre and Post-Operative Evaluation of Small Fenestra Stapedotomy in StapedialOtosclerosis.
}

\author{
AhamadNauphal Pullarat ${ }^{1}$,Ashish Chandra Agarwal ${ }^{2}$, Ramaswamy $^{3}$ \\ Balakrishnan $^{3}$, Kailesh Pujary ${ }^{4}$ \\ ${ }^{I}$ Assistant Professor, Department Of ENT, MES Medical College, PerinthalmannaMalappuram, Kerala, India. \\ ${ }^{2}$ Assistant Professor, Department Of ENT, Kasturba Medical College, Manipal, Manipal University, \\ Karnataka, India. \\ ${ }^{3}$ Professor AndHead, Department Of ENT, Kasturba Medical College, Manipal, Manipal University, \\ Karnataka, India. \\ ${ }^{4}$ Associate Professor, Department Of ENT, Kasturba Medical College, Manipal, Manipal University, \\ Karnataka, India.
}

\begin{abstract}
:
Context: Evaluation of hearing acuity after small fenestra stapedotomy for stapedialotosclerosis.

Aim: The aim of this study was to profile the age/ gender distribution, symptoms and hearing evaluation (before and after surgery) of patients diagnosed with otosclerosis.

Setting and design: The study was done at a tertiary care hospital. It was a cross sectional record based study.

Subject and Methods: Forty three patients diagnosed with stapedialotosclerosis who satisfied the selection criteria and consented were included in the study. Agel gender distribution, symptoms and hearing acuity (before and after surgery) were profiled.

Statistical analysis used: Statistical analysis was performed using Statistical Package for Social Sciences (SPSS) version 11.5 (SPSS: An IBM company). For the current study frequency and percentage were considered.

Results: Majority of the patients presented between the age of 21 and 40 years. There were more females than males in our study group, hearing loss was the most common symptom. Most of the patients had a pre-operative air-bone ( $A B)$ gap of 31-50 dB, which improved to less than $10 \mathrm{~dB}$ six months after surgery.

Conclusion: Small fenestra stapedotomy is a safe and effective surgery for the patients having stapedialotosclerosis.
\end{abstract}

Keywords:otosclerosis; small fenestra; stapedotomy

\section{Introduction}

Otosclerosis is an autosomal dominant disease of the otic capsule. It is characterized by alternate phases of bone resorption and formation. Mature lamellar bone is removed by osteoclasts and is replaced by woven bone of greater thickness, cellularity and vascularity. Fissula ante fenestram, an area lying in front of oval window, is the site of predilection for stapedialotosclerosis. If the bony changes are such that clinical manifestations are evident, the term clinical otosclerosisis used, otherwise it is called histological otosclerosis. Usually the patients have a conductive hearing loss, cochlear involvement leads to sensorineural component.

There are a number of studies in English literature on otosclerosis from across the developed world. This study was done with the aim to profile the disease among Indians, particularly those living along the west coast of Indian peninsula, to evaluate the results of surgery in the hands of experienced surgeons in conditions existing in a developing nation like India and to describe the complications arising as a result of surgery.

\section{Material And Methods}

A cross sectional record based study was donebetween January 2000 and December 2005 on 43 consecutive patients diagnosed with otosclerosis who underwent small fenestra stapedotomy at the department of otorhinolaryngology of a tertiary care hospital. Subjects included in the study were those who were clinically diagnosed to have otosclerosis and were found to have stapes foot-plate fixation during exploratory tympanotomy. All subjects of both the genders above the age of 12 years were included in the study. Patients with sensory-neural hearing loss, having a past history of ear surgery or any other external/ middle ear pathology, those who lost to follow-up in less than six months of surgery were excluded from the study. A clearance from the Institutional Ethics Committee (IEC) was obtained prior to the start of the study. Data relating to age and gender distribution, symptoms, audiometry and tympanometry findings, complicating factors, complications and length of piston (teflon) used was compiled and analyzed. 


\section{Results}

A total of 43 subjects (43 ears) were considered for the study, there were 17 males and 26 females. The age of the subjects varied from 20 to 55 years, the mean age was 32 years. Hearing loss was the most common symptom which was reported by all the subjects. Some of them also complained of tinnitus and vertigo. The analysis of symptoms is shown in Table 1. Table 2 shows the audiometry findings of the operated ear. Majority of the subjects $(51.2 \%)$ had a Pure Tone Average (PTA) between 56 and $70 \mathrm{~dB}$. The pre-operative air-bone (AB) gap is mentioned in Table 3. 32.5\% of the subjects had an AB gap between 31 and 40 dB.Tympanogram findings are analyzed in Table 4. Majority of the subjects (62.7\%) had a type 'A' tympanogram. A piston length ranging from 3.5 to $4.5 \mathrm{~mm}$ was used intra-operatively. Table 5 shows the pistonlength distribution across the subjects. Facial nerve abnormality was the commonest $(6.9 \%)$ complicating factor found intra-operatively. Persistent stapedial artery was found in $2.3 \%$ of the subjects. Vertigo was the most common (30.23\%) complaint of the subjects post operatively. The incidence of complications in our study is mentioned in Table 6 . There was a significant improvement in the AB gap 6 months after the surgery, with 38 subjects (88.3\%) having an $\mathrm{AB}$ gap of less than $10 \mathrm{~dB}$ and rest of them had it between 11 and $20 \mathrm{~dB}$.Table 7 shows the AB gap gain at the frequencies of 500, 1000 and $2000 \mathrm{~Hz}, 6$ months after surgery. The gain was maximum $(35.93 \mathrm{~dB})$ at $500 \mathrm{~Hz}$.

\section{Discussion}

In the present study 43 patients with otosclerosis who underwent small fenestra stapedotomywere assessed to profile the age/ gender distribution, symptoms and hearing evaluation (before and after surgery). The mean age of the subjects included in the study was 32 years, and there were more females as compared to males. The primary symptom was gradually progressive hearing loss. Tinnitus was seen in $65 \%$ of the patients. Goodhill $^{[1]}$ proposed that tinnitus in otosclerosis was a class of 'unmasked visceral tinnitus' which could be a feature of any pathology associated with conductive deafness and is due to normal sub audible tympanic/peritympanic vascular and muscular noises that are unmasked by the conductive deficit. $62.7 \%$ had a type ' $A$ ' tympanogram and $34.8 \%$ had 'As' type curve. A trans-canal approach was used to perform all the surgeries.Post-operatively $88.3 \%$ of the subjects had an AB gap within 10dBand rest had within $20 \mathrm{~dB}$ at 6 months. The mean post-operative Air- Bone gap was 9.41dB. The p value comparing pre and post-operative AB gap with paired-t test was $<0.001$. This difference is statistically significant. A study by Quaranta et al ${ }^{[2]}$ reported that $84.8 \%$ of small fenestra stapedotomies had a post-operative $\mathrm{AB}$ gap within $10 \mathrm{~dB}$ and the mean value was 6 $\mathrm{dB}$.

The post-operative $\mathrm{AB}$ gap at $500 \mathrm{~Hz}, 1000 \mathrm{~Hz}$ and $2000 \mathrm{~Hz}$ was compared to the pre-operative $\mathrm{AB}$ gap at these three frequencies. The mean $\mathrm{AB}$ gap gain at frequency $500 \mathrm{~Hz}$ was $35.93 \mathrm{~dB}$, at $1000 \mathrm{~Hz}$ was 31.75 $\mathrm{dB}$ and at $2000 \mathrm{~Hz}$ was $23.65 \mathrm{~dB}$. The gain was better at low frequencies. On comparing the pre and postoperative $A B$ gap with paired-t test, the value was statistically significant $(p<0.017)$. The results of this study can be compared to the study by Hiromi Ueda et al ${ }^{[3]}$, who reviewed 83 patients with small fenestra stapedotomy. He concluded that closure of $\mathrm{AB}$ gap after stapes surgery is poor below $1000 \mathrm{~Hz}$ and above 8000 $\mathrm{Hz}$, while it is good at $2000 \mathrm{~Hz}$ and $4000 \mathrm{~Hz}$. This is important for good speech understanding.

In this study, vertigo was the most common complaint after surgery, with $30.23 \%$ of the subjects reporting it. $11.62 \%$ of individuals reported altered taste and $9.3 \%$ developed sensory-neural hearing loss. There was tympanic membrane tear in one case. Palva et al ${ }^{[4]}$ reported, in a study of 456 ears (360 patients), three ear drum perforations- one infected and two dry, perilymph fistula in 7 and sensorineural hearing loss in 6 individuals. Salvinelli et al ${ }^{[5]}$ reported delayed facial palsy in 7 out of 706 stapedectomies. They proposed a viral origin and suggested prophylactic therapy with acyclovir during the whole perioperative period.Massey et $\mathrm{al}^{[6]}$ reported sensorineural hearing loss in $2.8 \%$ subjects after stapedectomy. Lippy et al ${ }^{[7]}$ had 2 cases of sensorineural hearing loss and 6 cases of tympanic membrane tear.

The use of computed tomography scan of temporal bone, though taxing to the patient, has been quite an effective tool in the pre-operative planning. It cautions the surgeon of problems that may be encountered during the surgery like congenital dehiscence of the facial canal and thick footplate due to obliterativeotosclerosis. In cases of failed surgery, a scan can show displacement of the piston from the fenestra.

\section{Conclusions}

Though the sample size considered in this study was small, the study offers significant clinical implications. The results support the role of small fenestra stapedotomy as a primary treatment for otosclerosis with maximal effectiveness and minimal complications. Mean post-operative Air - Bone gap closure following small fenestra stapedotomyafter 6 months was $9.41 \mathrm{~dB}$ which substantiates that this surgery is an ideal procedure for correcting the conductive component of hearing loss in otosclerosis. 


\section{References}

[1]. Goodhill V. The use of cortisone in otosclerosis. Trans Am AcadOphthalmolOtolaryngol. 1952 Jul-Aug;56(4):635-46.

[2]. Quaranta N, Besozzi G, Fallacara RA, Quaranta A.Air and bone conduction change after stapedotomy and partial stapedectomy for otosclerosis.Otolaryngol Head Neck Surg. 2005 Jul;133(1):116-20.

[3]. Ueda H, Miyazawa T, Asahi K, Yanagita N. Factors affecting hearing results after stapes surgery. J Laryngol Otol. 1999 May;113(5):417-21.

[4]. Palva T, Karja J, Palva A.Otosclerosis surgery. ActaOtolaryngol. 1977 Mar-Apr;83(3-4):328-35.

[5]. Salvinelli F, Casale M, Trivelli M, Di Peco V, Greco F. Otosclerosis and stapedoplasty in older adults. J Am Geriatr Soc. 2002 Aug;50(8):1396-400.

[6]. Massey BL, Kennedy RJ, Shelton C. Stapedectomy outcomes: titanium versus teflon wire prosthesis. Laryngoscope. 2005 Feb;115(2):249-52.

[7]. Lippy WH, Berenholz LP, Burkey JM. Otosclerosis in the 1960s, 1970s, 1980s, and 1990s. Laryngoscope. 1999 Aug;109(8):13079. 